
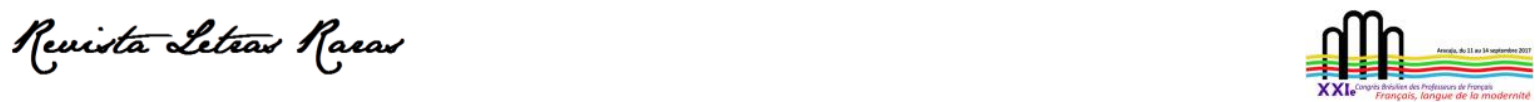

Actes du XXlème Congrès Brésilien des Professeurs de Français dans Édition spéciale de la Revue Letras Raras, 2021

ISSN : 2317-2347 - v. 10, Dossier Spécial (2021)

Todo o conteúdo da RLR está licenciado sob Creative Commons Atribuição 4.0 Internacional

\title{
L'évaluation formative en français langue étrangère : le portfolio et l'écriture réflexive en contexte universitaire
}

\section{Denise Gisele de Britto Damasco}

Licenciée en Lettres (1986), ayant un Master Recherche (2008) et Doctorat (2014) en Éducation à I'Université de Brasilia. Enseignante de français langue étrangère au Secrétariat d'État d'Éducation du DF de 1989 à 2015. Actuellement, suit des études postdoctorales à la PUC SP et préside la Fédération brésilienne des professeurs de français (2017-2020 et 2020/2022).

iD http://orcid.org/0000-0002-0250-0776

Reçu en : 23 août 2021. Approuvé en: 09 septembre 2021.

\section{Comment citer cet article:}

DAMASCO, Denise. L'évaluation formative en français langue étrangère : le portfolio et l'écriture réflexive en contexte universitaire. Revista Letras Raras, p. 230-242, n. Spécial, v. 10, nov. 2021.

\section{RÉSUMÉ}

Cette communication présente une expérience didactique menée en 2016 sur l'évaluation formative des apprentissages en Français Langue Étrangère - FLE à l'Institut de Lettres d'une université publique brésilienne avec des futurs enseignants de français, professionnels en Langues Etrangères Appliquées (LEA) et traducteurs. Nous essayerons de comprendre le sens de l'évaluation formative à partir de Villas Boas $(2002 ; 2005 ; 2008 ; 2017 ; 2019)$ et Veltcheff (2009) ainsi que la mise en place des portfolios et des synthèses réflexives, une pratique d'écriture quotidienne en classe. La question principale de cette recherche est : Comment comprendre l'usage des portfolios et de l'écriture réflexive dans le cadre de l'évaluation formative en contexte universitaire ? Une autre question se pose : comment les étudiants ont-ils réagi à ces deux façons d'évaluer ? Les données de cette recherche ont été obtenues à partir de l'analyse des synthèses réflexives présentées et des portfolios rendus en fin de semestre. Nous constatons que l'évaluation formative a facilité le dialogue et la communication entre l'enseignant et l'apprenant et qu'elle a induit des moments d'apprentissage sur la langue française, surtout sur sa structure morphosyntaxique et sur la pratique d'évaluation formative en cours de langues.

MOTS-CLÉS : Évaluation formative ; Enseignement du français langue étrangère ; Portfolio ; Synthèse réflexive ; Formation initiale.

\section{Introduction}

Cette communication présente une expérience menée en 2016 sur l'évaluation formative des apprentissages en Français Langue Etrangère - FLE à l'Institut de Lettres d'une université publique brésilienne avec des futurs enseignants, professionnels en Langues Etrangères Appliquées (LEA) et traducteurs. La question principale de cette recherche est : Comment 

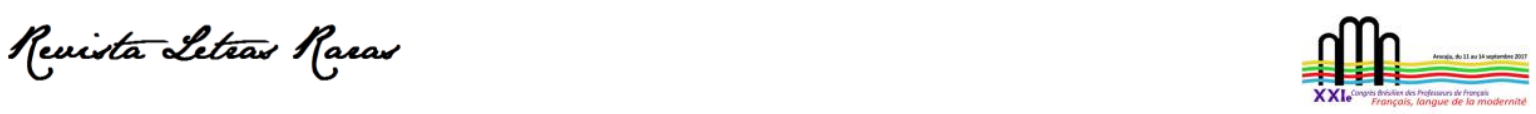

Actes du XXlème Congrès Brésilien des Professeurs de Français dans Édition spéciale de la Revue Letras Raras, 2021 ISSN : 2317-2347 - v. 10, Dossier Spécial (2021)

Todo o conteúdo da RLR está licenciado sob Creative Commons Atribuição 4.0 Internacional

comprendre l'usage des portfolios et de l'écriture réflexive dans le cadre de l'évaluation formative ? Une autre question se présente : comment les étudiants ont-ils réagi à ces deux façons d'évaluer ? Comme écriture réflexive nous avons choisi d'utiliser la synthèse réflexive comme un « retour sur l'action journalière » (CHANTAL, 1998). Le matériel du premier semestre a été analysé afin d'améliorer les pratiques ainsi que pour peaufiner les actions et le programme du cours au second semestre. Les données de cette recherche ont été obtenues à partir de l'analyse des synthèses réflexives et des portfolios rendus à la fin du second semestre de 2016 qui a vraiment été clôturé fin janvier 2017.

Comme point de départ pour la discussion, nous considérons que la didactique de langues nous propose des approches communicatives et actionnelles afin de mettre l'emphase sur les fonctions sociales de l'utilisation de la langue et que l'enseignement de langues n'est pas seulement l'enseignement d'un système linguistique. Cette expérience didactique a été rapportée oralement en 2017 lors de trois congrès scientifiques internationaux. Au Brésil, lors de la XXlème édition du congrès brésilien des professeurs de français. Au Canada, lors du IV Colloque international en éducation, organisé par le Centre de Recherche sur la Formation et la Profession Enseignante (CRIFPE) et aussi lors d'un colloque organisé dans le cadre de l'ACFAS, à Montréal. En 2018, nous avons eu l'occasion de revivre cette expérience lors du premier congrès international de formation enseignante du CRIFPE à Santiago, au Chili. En 2020, lors de la IVe Rencontre en Français et de la XXle Journée de Formation, dont le thème proposé était: Interactions et collaboration en ligne : les potentialités des outils numériques pour l'enseignement et l'apprentissage du FLE/FLS organisées par l'Université de São Paulo et de l'Association des Professeurs de Français de São Paulo, nous avons pu reprendre cette expérience et réfléchir ensemble et en atelier sur des pratiques d'évaluation formative dans l'enseignement du français. Cela nous montre que la thématique liée à l'évaluation est toujours vivante et motivante pour les enseignants et pour la formation enseignante. L'évaluation est une activité intrinsèque de l'enseignant et même si « longtemps cantonnée dans son propre domaine [...], l'évaluation est devenue, au cours de la décennie 1990, l'une des préoccupations majeures de la didactique des langues étrangères et, en particulier, du Français langue étrangère - FLE » (CUQ, GRUCA, 2017, p. 201). 


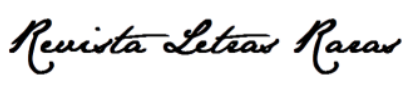

Actes du XXlème Congrès Brésilien des Professeurs de Français dans Édition spéciale de la Revue Letras Raras, 2021 ISSN : 2317-2347 - v. 10, Dossier Spécial (2021)

Todo o conteúdo da RLR está licenciado sob Creative Commons Atribuição 4.0 Internacional

\section{2 Évaluation formative : comment et pourquoi ?}

Nous croyons que pour les futurs enseignants éveiller le sens et l'étude de l'évaluation formative à partir des expériences didactiques vécues en cours de formation initiale, soit en présentiel, soit à distance, est incontournable dans la formation enseignante. Selon Hedid (2017), " les futurs enseignants ne sont pas formés à évaluer, mais à établir des bilans pour synthétiser : c'est le constat que nous relevons, à chaque fois, suite à la lecture des programmes de formations » (HEDID, 2017, p. 111). Pour cette chercheuse, nous constatons dans la pratique de plusieurs enseignants une sorte de reprise de ce que leurs prédécesseurs faisaient. D'après l'auteure, "dans leur pratique, ils essayent d'imiter leurs anciens enseignants, de jouer aux apprentis évaluateurs sans avoir pour autant le matériel adéquat »(HEDID, 2017, p. 111).

Nous comprenons que l'évaluation est un moment d'apprentissage et non de contrôle ou de punition (VILLAS BOAS, 2002 ; 2005 ; VELTCHEFF, 2009). Cet article ne vise pas à définir tous les types d'évaluation, mais de présenter une expérience didactique à partir de l'évaluation formative. Nous considérons que l'évaluation formative propose des pratiques qui dépassent l'idée de classer, sélectionner, punir, faire redoubler, afin de mettre en place une évaluation positive (VILLAS BOAS, 2002 ; 2005 ; 2008). Selon Villas Boas (2008), l'évaluation formative nous mène à encourager la conception de l'erreur comme inhérente à l'apprentissage. D'après cette auteure, l'autonomie chez l'apprenant est fondamentale pour préparer de futurs enseignants et le fait de motiver les apprenants les fait avancer tous seuls en autonomie. Cette chercheuse nous apprend que proposer une évaluation comme un processus et non seulement comme un examen de fin de cours induit à une pédagogie de la réussite. Nous faisons progresser les étudiants. Comme contrainte, nous avons l'attribution d'une note.

Pour bien entamer une évaluation formative, il nous faut avoir des objectifs définis et faisables. C'est une évaluation positive, procédurale, qui valorise le savoir acquis et qui laisse des traces d'apprentissage. Ce n'est pas un examen de fin de cours. Nous ne sommes pas dans le cadre d'une obtention d'un certificat, bien au contraire, nous sommes en cours pour apprendre et pour réussir (VILLAS BOAS, 2002 ; $2005 ; 2008 ; 2017 ; \quad 2019$; VELTCHEFF, 2009). 

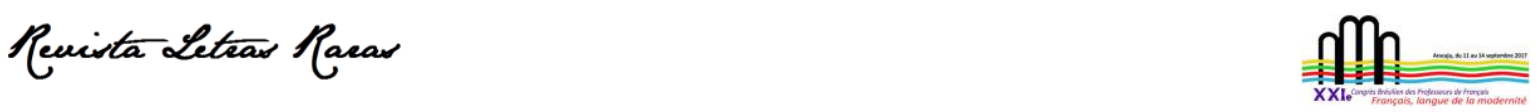

Actes du XXlème Congrès Brésilien des Professeurs de Français dans Édition spéciale de la Revue Letras Raras, 2021

ISSN : 2317-2347 - v. 10, Dossier Spécial (2021)

Todo o conteúdo da RLR está licenciado sob Creative Commons Atribuição 4.0 Internacional

Selon Villas Boas (2008) passer un test écrit peut devenir un instrument d'évaluation formative si l'apprenant a l'occasion de progresser. Alors, faire passer un test sur consultation peut induire à la reprise des contenus étudiés, aboutissant à l'apprentissage. La mise en place des portfolios et surtout des synthèses réflexives peut, donc, renvoyer à une pratique d'écriture quotidienne en classe. Le portfolio induit à la réflexion. Et il est important de saisir que c'est l'apprenant qui construit son portfolio. II prend des décisions et réfléchit sur ses décisions. Selon Vanhulle (2009), " l'usage du portfolio fait partie des dispositifs susceptibles de stimuler ce développement à travers la pratique de ce que nous nommerons un 'genre réflexif' »(VANHULLE, 2009, p. 202).

Castellotti (2008) nous explicite l'intérêt des portfolios et des approches biographiques pour les enfants dans un chapitre lié au rapport entre l'école française et les langues des enfants à travers la mobilisation de parcours plurilingues et pluriculturels. Selon cette chercheuse, « les portfolios sont une forme de matériel didactique s'inscrivant dans les approches biographiques pour en exploiter, de façon plus ciblée, une dimension éducative-réflexive accentuée » (CASTELLOTTI, 2008, p. 274). Elle nous confirme que l'utilisation du portfolio est intéressante à plusieurs niveaux puisque « ce type d'activité conduit à la valorisation du déjà-là (linguistique, scolaire, disciplinaire, social) [...] (CASTELLOTTI, 2008, p. 275).

Non seulement chez les enfants les portfolios peuvent contribuer dans l'apprentissage des langues, mais aussi chez les étudiants de l'enseignement supérieur, ce qui est le cas de ce rapport d'expérience didactique. Ambrósio (2013) approfondit sur l'usage du portfolio dans l'enseignement supérieur, en considérant cet outil comme

un outil pédagogique utilisé pour une collection organisée et planifiée de travaux produits par les étudiants, le long d'une période déterminée, de façon à pouvoir induire une vision élargie et détaillée de l'apprentissage réalisé, ainsi que les visions de différentes composantes de son développement cognitif, métacognitif et affectif. (AMBRÓSIO, 2013, p. 23, notre traduction) $)^{1}$

La pratique réflexive est une thématique largement approfondie surtout le fait de réfléchir sur sa pratique : Est-ce qu'il y a une meilleure façon de l'exécuter ? D'apprendre ? D'enseigner? Selon Tardif, Borges et Malo (2012) ce qui est difficile, c'est faire le passage du slogan

\footnotetext{
1 Traduction de: "[...] ferramenta pedagógica para uma coleção organizada e planejada de trabalhos produzidos pelos(as) estudantes, ao longo de um determinado período de tempo, de forma a poder proporcionar uma visão alargada e detalhada da aprendizagem efetuada bem como dos diferentes componentes do seu desenvolvimento cognitivo, metacognitivo e afetivo". (AMBRÓSIO, 2013, p. 23)
} 


\section{Reuista Letras Racas}

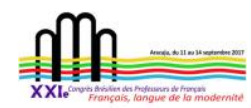

Actes du XXlème Congrès Brésilien des Professeurs de Français dans Édition spéciale de la Revue Letras Raras, 2021 ISSN : 2317-2347 - v. 10, Dossier Spécial (2021)

Todo o conteúdo da RLR está licenciado sob Creative Commons Atribuição 4.0 Internacional

pédagogique à la réflexion quotidienne. L'acte d'écrire nos réflexions au quotidien peut donc nous faire comprendre nos défis, nos limites, nos avancements et également la meilleure façon de réussir.

Hess (2005) nous propose dans son œuvre Le journal des idées un éventail de possibilités d'écritures, plusieurs formes de journaux, tels que : le journal intime; le journal de voyage ; le journal philosophique, celui de ses propres idées ; le journal de recherche ; le journal de formation ; le journal institutionnel, soit disant dans un hôpital par exemple ; l'écriture collective ; journal de moments : pour parler de la vie, des moments choisis ; et le journal onirique : un journal des songes. Selon ce chercheur, « Le journal est un outil efficace pour celui qui veut comprendre sa pratique, la réfléchir, l'organiser, la changer, la rendre cohérente avec ses idées » (HESS, 2005, p. 5). Un journal est tenu au jour le jour. Cette forme d'écrit personnel est inscrite dans le présent et dans un premier temps, le journal est écrit pour soi, mais c'est un écrit pour l'autre (HESS, 2005). Nous avons constaté qu'en formation, le journal est un outil de construction de compétences professionnelles. Ce qui est incontournable, c'est le fait d'écrire quotidiennement, comme une habitude, une réflexion qui devient habituelle à partir du retour du soi. Selon Coracini (2010), nous pouvons inciter et créer des situations pour écrire. Elle nous affirme que « il faut que l'élève ait la possibilité de se dire, au-delà de dire, de s'exposer, au-delà d'exposer pour qu'il vive cette expérience d'étrangéité (unheimlichkeit), la construction de soi, d'une identité illusoire, à vrai dire, nécessaire [...] (CORACINI, 2010, p. 46, notre traduction)2.

La synthèse réflexive a été l'instrument choisi pour évaluer l'écriture au jour le jour. Cela a été fait à partir des thèmes discutés pendant les séances qui ont eu lieu durant les deux cours de la Morphosyntaxe du français.

\section{Contexte de cette expérience didactique}

Cette expérience a eu lieu dans le cadre d'une discipline de morphosyntaxe du cours de Lettres françaises dans une université publique brésilienne en 2016. II faut souligner que pendant deux semestres consécutifs nous avons fait cette pratique d'évaluation formative.

\footnotetext{
${ }^{2}$ Traduction de : «É preciso, portanto, que o aluno tenha a possibiliade de se dizer, mais do que dizer, de se expor, mais do que expor para que vivencie a experiência do estranhamento (unheimlichkeit), a construção de si, de uma identidade ilusória [...]" (CORACINI, 2010, p. 46).
} 

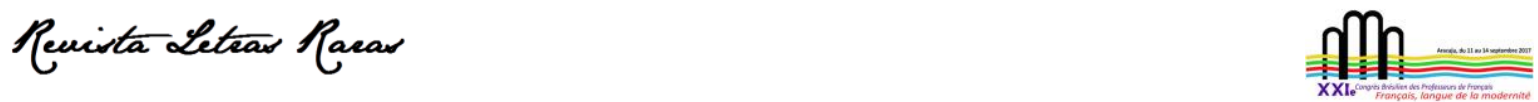

Actes du XXlème Congrès Brésilien des Professeurs de Français dans Édition spéciale de la Revue Letras Raras, 2021 ISSN : 2317-2347 - v. 10, Dossier Spécial (2021)

Todo o conteúdo da RLR está licenciado sob Creative Commons Atribuição 4.0 Internacional

Le premier semestre a duré de mars à juillet avec un groupe composé de onze étudiants et, au second semestre, de trente étudiants, ayant un calendrier différencié dû à une grève des étudiants de cette université. Alors, le cours a commencé en mars, mais en octobre les activités se sont arrêtées. Nous les avons reprises fin décembre jusqu'en janvier.

Le déroulement des séances et les activités notées en Morphosyntaxe du français ont eu lieu pendant les 30 rencontres en présentiel et deux fois par semaine. Chaque séance avait un volume horaire de 100 minutes et était rythmée à partir d'un exposé de l'enseignante sur le thème proposé entre 20 et 30 minutes, ensuite nous proposions aux étudiants soit une activité collective, soit en binôme ou individuelle ayant également un volume horaire de 20 ou 30 minutes. À la fin de la séance, nous nous arrêtions pour écrire, de façon individuelle une synthèse réflexive. Afin d'accomplir ce programme, intitulé officiellement ementa du cours, nous discutions au préalable les détails des activités ainsi que les étapes des évaluations, comme la durée et l'organisation des séances, entre autres. C'était notre contrat d'apprentissage qui devenait un engagement collectif et individuel. Au moment de discuter le programme du cours, nous induisons aux premiers dialogues, nous pouvions faire nos accords, commenter les suggestions des étudiants et leurs doutes.

Dans le cadre de notre proposition d'évaluation formative, nous prévoyions comme instruments d'évaluation des tests écrits et un test supplémentaire pour ceux et celles ayant des problèmes d'absence ; des synthèses réflexives ; une présentation orale/un exposé oral en classe ; une publication en ligne dans la plateforme officielle du cours, intitulée Moodle et un portfolio à rendre à la fin du semestre. L'université avait un système de notation semestrielle obligatoire de 100 points. La note dans la classe devient le thème des réflexions de Bugnard (2015) qui analyse les enjeux liés à l'attribution d'une note à un étudiant. Cela n'est pas la thématique de notre article. Alors, nous avons proposé lors de ces deux semestres la notation suivante selon le tableau 1 :

Tableau 1 : Notation proposée dans le cadre de cette expérience.

\begin{tabular}{|l|c|c|}
\hline \multicolumn{1}{|c|}{ Instrument d'évaluation } & $\begin{array}{c}\text { Nombre d'évaluation proposée par } \\
\text { semestre }\end{array}$ & Notation totale sur 100 \\
\hline Test écrit & 3 & 30 points \\
\hline $\begin{array}{l}\text { Présentation sur un espace } \\
\text { numérique }\end{array}$ & 1 & 10 points \\
\hline Synthèse réflexive & 23 & 20 points \\
\hline $\begin{array}{l}\text { Activité notée pour la préparation du } \\
\text { portfolio }\end{array}$ & 2 & 10 points \\
\hline
\end{tabular}



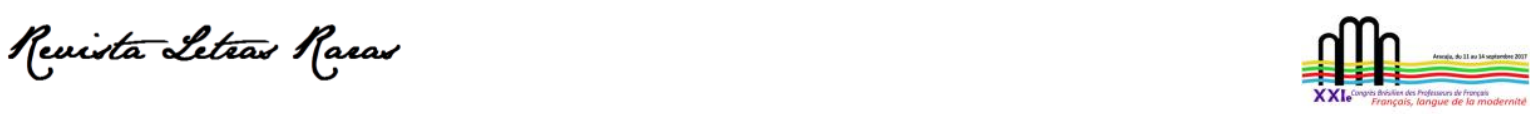

Actes du XXlème Congrès Brésilien des Professeurs de Français dans Édition spéciale de la Revue Letras Raras, 2021 ISSN : 2317-2347 - v. 10, Dossier Spécial (2021)

Todo o conteúdo da RLR está licenciado sob Creative Commons Atribuição 4.0 Internacional

\begin{tabular}{|l|l|l|}
\hline Portfolio & 1 & 30 points \\
\hline
\end{tabular}

Source : Tableau élaboré en 2017 pour la présentation orale lors du XXI CBPF à Aracaju.

Les tests écrits ont été proposés sur consultation pour que cela ouvre un espace d'apprentissage. Nous avons proposé, lors de ces deux semestres, trois tests écrits et un test à ceux et celles ayant des problèmes personnels justifiés officiellement. Alors, nous avons préparé quatre tests le long de ces semestres. Ceux et celles ayant obtenu une note pas favorable ont eu la possibilité de passer le quatrième test comme un test de rattrapage, un recours permettant de changer la note. Quant aux synthèses réflexives, nous leur avons proposé un point par synthèse et tout au long de ces semestres, nous avons réalisés environ 23 synthèses. Synthèse écrite et rendue, un point. Alors, les étudiants ont eu la possibilité d'avoir 20 points en 23 possibilités de réalisation de synthèse. Quant à la publication sur la plateforme Moodle, l'espace numérique prévu, c'était un libre choix de ce qu'il fallait réaliser : soit écrit, soit oral. Nous avons prévu une séance pour faire connaissance de ces activités en ligne. Nous avons eu des étudiants qui ont écrit des poèmes, qui ont pensé à une activité didactique à partir d'un document authentique, nous avons eu des capsules-vidéos, entre autres. Puis, en ce qui concerne le portfolio, nous avons valorisé les activités de préparation de cet outil (10 points) et le produit final rendu (30 points). Pour préparer leur portfolio, nous avons prévu deux séances et une activité par séance. La présence aux activités ainsi que la participation aux séances valaient la notation prévue.

Nous avons observé que les doutes concernant le portfolio et la synthèse réflexive surgissaient au fur et à mesure que la discipline se déroulait. Au départ, il y avait une hésitation avant d'écrire la synthèse réflexive proposée par séance, mais la présence d'un moniteur en cours nous aidait à répondre aux demandes des étudiants. Un retour au programme et sa relecture ont été les stratégies menées pour répondre aux questions des participants. Nous nous sommes rendu compte que, malgré la présentation, la discussion et les commentaires du programme réalisés lors des premières séances n'étaient pas suffisants pour que les étudiants comprennent le schéma d'évaluation proposé.

Les synthèses réflexives devraient démontrer les pensées individuelles issues des discussions menées et des thèmes abordés dans les cours de la Morphosyntaxe du français. Pour cela, nous posions une question pour déclencher la réflexion. Le fait d'écrire en classe le long de 23 séances pendant 20 et 30 minutes a entamé une nouvelle routine en cours. L'usage du 

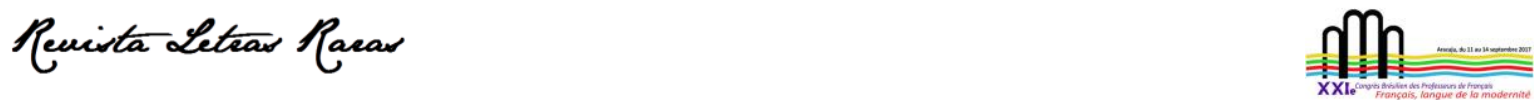

Actes du XXlème Congrès Brésilien des Professeurs de Français dans Édition spéciale de la Revue Letras Raras, 2021 ISSN : 2317-2347 - v. 10, Dossier Spécial (2021)

Todo o conteúdo da RLR está licenciado sob Creative Commons Atribuição 4.0 Internacional

dictionnaire était permis. À la fin, les apprenants rendaient un brouillon s'ills n'avaient pas eu le temps de le mettre au propre. Quelques-uns le faisaient par courriel pendant le cours. Pour les apprenants absents en cours, ce n'était pas possible de réaliser la synthèse réflexive prévue. La solution proposée, en guise de notation à la fin du cours, a été celle de compter 20 réflexions sur un total de 23 synthèses, dont 20 points pour ceux et celles ayant rendu leurs synthèses. Nous rappelons que cette discipline prévoyait 30 séances. Alors, il fallait faire cette activité d'écriture en classe, obligatoirement, sous forme d'un paragraphe à partir d'une question proposée par l'enseignante. La rétroaction de l'enseignante avait lieu dans la séance suivante, donnant la possibilité aux apprenants de la réécrire. Ce cycle vécu : écrire, recevoir une rétroaction, réécriture, relecture de l'enseignante a rythmé l'année 2016 dans le cadre de la discipline de la Morphosyntaxe.

\section{Analyse et discussion}

L'expérience menée lors du premier semestre de 2016 nous a fait mieux organiser et schématiser notre pratique enseignante par rapport à l'évaluation formative. Alors, nous avons décidé d'analyser les synthèses réflexives et les portfolios qui ont été rendus pendant le cours réalisé durant le second semestre de 2016, ayant 30 étudiants inscrits. Selon le tableau 2, nous vous proposons une analyse du contenu des synthèses réflexives rendues et lues par l'enseignante.

Tableau 2 : Synthèses réflexives proposées.

\begin{tabular}{|l|l|c|}
\hline Catégorie d'analyse & \multicolumn{1}{|c|}{ Thèmes proposés par catégorie } & $\begin{array}{c}\text { Total de thèmes par } \\
\text { catégorie }\end{array}$ \\
\hline Evaluation formative & $\begin{array}{l}\text { Evaluation positive / Synthèse réflexive / Portfolio / Auto-évaluation } \\
\text { / Commentaires des tests écrits / Grille d'évaluation }\end{array}$ & 6 \\
\hline $\begin{array}{l}\text { La langue et la } \\
\text { grammaire }\end{array}$ & $\begin{array}{l}\text { Représentation de langue et grammaire / Types de grammaire / } \\
\text { Distinguer la morpho de la syntaxe / Les procédés de l'analyse } \\
\text { logique de la phrase / Phrases simples ou complexes }\end{array}$ & 5 \\
\hline $\begin{array}{l}\text { Apprentissage du } \\
\begin{array}{l}\text { Français langue } \\
\text { étrangère (FLE) }\end{array}\end{array}$ & $\begin{array}{l}\text { Les prépositions / Les verbes / Le discours rapporté / La } \\
\text { ponctuation / La nominalisation }\end{array}$ & 5 \\
\hline $\begin{array}{l}\text { Activités hors cours } \\
\text { et l'imprévu en } \\
\text { classe }\end{array}$ & $\begin{array}{l}\text { Bibliothèque de cette université / Conférence de la Francophonie } \\
\text { Canadienne / La grève et les occupations des espaces de } \\
\text { l'Université par des étudiants.es }\end{array}$ & 3 \\
\hline $\begin{array}{l}\text { Récits de soi } \\
\text { Ce plaisir d'apprendre / Travailler en groupe ou individuellement / } \\
\text { Communiquer / Projets d'avenir }\end{array}$ & 4 \\
\hline
\end{tabular}

Source : Tableau élaboré en 2017 pour la présentation orale lors du XXI CBPF à Aracaju. 

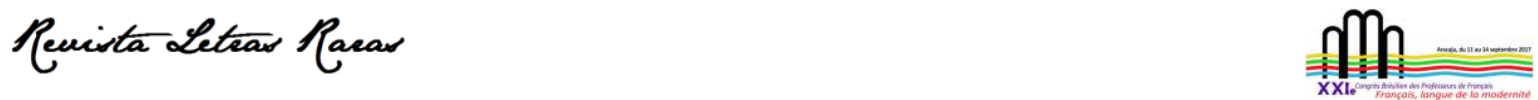

Actes du XXlème Congrès Brésilien des Professeurs de Français dans Édition spéciale de la Revue Letras Raras, 2021 ISSN : 2317-2347 - v. 10, Dossier Spécial (2021)

Todo o conteúdo da RLR está licenciado sob Creative Commons Atribuição 4.0 Internacional

Nous avons recueilli plusieurs témoignages des apprenants pendant les deux semestres de ce cours de Morphosyntaxe du français. Quand nous leur avons demandé leur perception sur l'activité relative aux synthèses réflexives et sur leurs synthèses écrites et rendues, nous avons obtenu, par exemple, comme réponse : "La synthèse est une production plus objective que le journal de formation parce qu'elle est une réponse à une question » (étudiant $A$, le 8/8/2017). Nous avons compris que ces apprenants avaient vécus d'autres expériences d'écriture quotidienne et qu'ils sont arrivés à distinguer le journal de formation et la synthèse réflexive.

Nous aimerions souligner que pendant le second semestre de 2016, ce groupe, composé par 30 apprenants, a écrit au total 448 synthèses réflexives qui ont été lues et corrigées par l'enseignante. Le graphique 1 nous démontre cela.

Graphique 1 : Total de synthèses réflexives rendues par semaine lors du second semestre de 2016.

\section{Nombre de Synthèses Réflexives par séance}

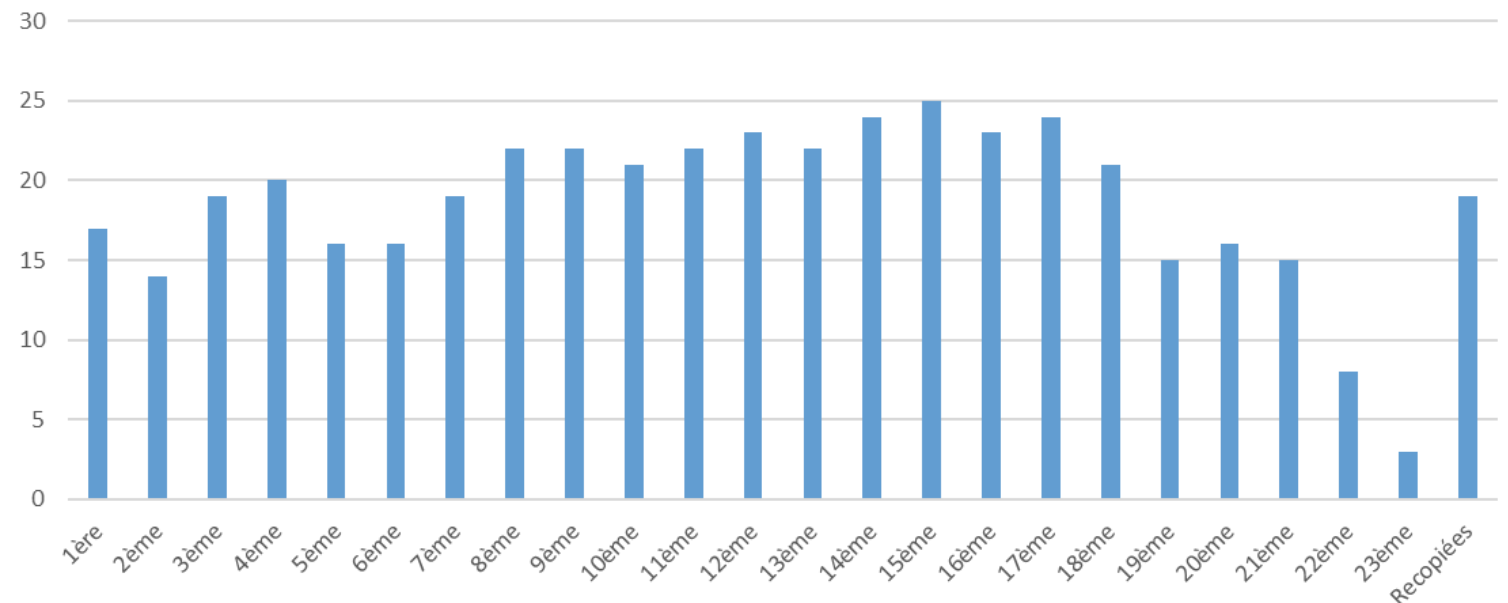

Source : Tableau élaboré en 2017 pour la présentation orale lors du XXI CBPF à Aracaju.

Nous constatons qu'il y a eu moins de synthèses rendues au début et à la fin du cours. Nous considérons qu'au début du cours, c'est normal puisque cette démarche était nouvelle pour la plupart des apprenants. À la fin du cours, à cause d'une grève, le cours a été repris après la 18ème séance, ce qui a rendu difficile la reprise du rythme d'études en fin d'année, après une longue période de grève et d'occupation des espaces de l'Université par les étudiants. Nous observons 

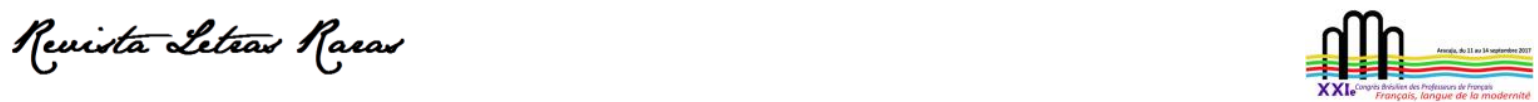

Actes du XXlème Congrès Brésilien des Professeurs de Français dans Édition spéciale de la Revue Letras Raras, 2021 ISSN : 2317-2347 - v. 10, Dossier Spécial (2021)

Todo o conteúdo da RLR está licenciado sob Creative Commons Atribuição 4.0 Internacional

également qu'environ une vingtaine de synthèses ont été recopiées et recorrigées, ce qui montre que la plupart des apprenants ont jugé important la réécriture de leurs synthèses.

Quant au portfolio, nous avons ressenti chez les apprenants un sentiment allant de l'inquiétude à la satisfaction. À l'époque, le portfolio était une pratique assez récente chez les apprenants et futurs enseignants. Ce qui continue de l'être, selon nous. Nous avons reçu à la fin des cours deux types de portfolio, faisant un total de 27 portfolios pendant le second semestre : a) portfolios sur papier et b) portfolios numériques. Les portfolios sur papier avaient été organisés comme des archives dans des chemises avec des feuilles plastiques. D'autres ressemblaient à des livres, à des catalogues, à un mémoire de recherche et nous avons reçu un portfolio qui avait la forme d'une brochure géante. Le jour de la présentation de ce portfolio, type brochure ou dépliant, l'étudiante s'est déplacée dans l'espace de la salle et le groupe a ouvert son portfolio ensemble. La créativité était émergente et constatée à travers également des couvertures des portfolios. II y a eu une mosaïque faite à partir des journaux et une couverture en tissu et brodée. Il y a eu des portfolios rendus dans des boîtes et cartons. Dans les pages internes des portfolios, nous avons observé la présence de post-it pour attirer l'attention sur des activités importantes. Nous avons eu un portfolio rendu qui avait été préparé comme un catalogue de musée. Les portfolios numériques avaient été proposés comme un blog, comme des réflexions sur le réseau social Twitter et sur la plateforme Padlet, un mur.

Nous avons constaté qu'à la fin du cours les portfolios rassemblaient tout ce qui avait été significatif pour l'apprenant. Les séances préparatoires réalisées auparavant ont été fondamentales pour la discussion et la réflexion autour du choix du type de portfolio. Nous avons conclu que deux séances pour cette préparation ont été suffisantes et, pendant ces deux séances, nous avons eu le partage des décisions prises. Selon Vanhulle (2009), le portfolio fait réfléchir et « seul un développement réflexif véritable peut permettre au futur enseignant de se construire une culture professionnelle pertinente mais aussi distanciée et critique par rapport à ses attentes et savoirs »(VANHULLE, 2009, p. 202).

Nous avons observé qu'il y a eu également une personnification du portfolio. Les apprenants ont communiqué à travers leur portfolio. Une étudiante l'a appelé « mon petit » et elle a aussi dessiné son image tout au long du portfolio. Un autre étudiant a invité un futur lecteur du portfolio: "Ouvre-moi ». D'autres apprenants sont allés plus loin. Ils ont ajouté dans leurs portfolios des schémas d'études travaillés en cours et des textes étudiés en dehors de tout ce que 


\section{Reuista Letras Pacas}

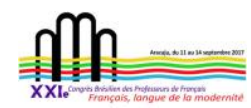

Actes du XXlème Congrès Brésilien des Professeurs de Français dans Édition spéciale de la Revue Letras Raras, 2021 ISSN : 2317-2347 - v. 10, Dossier Spécial (2021)

Todo o conteúdo da RLR está licenciado sob Creative Commons Atribuição 4.0 Internacional

nous avions fait tout au long du semestre. À la fin du semestre, la mise en commun des portfolios et le partage de cette expérience commune en évaluation formative ont été très enrichissants. Selon Lima (2003), l'étudiant construit son image en tant qu'apprenant à partir de toute sorte d'indication en salle de classe. II arrive à s'apprécier dans son contexte d'apprentissage, une conséquence et un effet de l'évaluation que nous discutons rarement (LIMA, 2013). Pour cette auteure, « toute sorte d'expérience à l'école, de réussite ou d'échec, composera le processus de développement de l'apprenant »(LIMA, 2013, p. 4, notre traduction) ${ }^{3}$.

\section{Considérations finales}

L'évaluation formative n'a de sens que par rapport aux objectifs d'apprentissages visés. Les spécialistes en FLE rejoignent les spécialistes en pédagogie pour mieux comprendre le sens de l'évaluation formative en cours. Selon Cuq et Gruca,

L'évaluation reste avant tout un acte formatif, qui se situe dans le continuum de l'apprentissage et de l'enseignement et qui apporte une contribution importante à l'appropriation d'une langue étrangère. Évaluer ne doit pas être une fin mais un moyen. (CUQ ; GRUCA, 2017, p. 213)

Faire écrire pour réfléchir était le moyen pour atteindre l'objectif majeur dans la discipline de Morphosyntaxe du français : apprendre la grammaire du français.

Malgré le fait de constater que ces étudiants n'avaient pas reconnu, au départ, le portfolio comme une pratique d'évaluation formative, comme cette pratique d'évaluation valorise le processus, nous croyons avoir atteint notre but quand ces étudiants ont exprimé leur satisfaction à les accomplir.

Nous pouvons conclure que les échanges entre l'enseignante et les étudiants ont eu lieu à partir de la lecture de leurs synthèses réflexives et de leur portfolio. L'évaluation formative à partir de ces deux instruments a progressivement facilité la communication en cours. Cela a été la thématique d'une présentation de cette expérience lors de l'événement Acfas ${ }^{4}$, en 2017 : communiquer à partir de l'évaluation. Les tests écrits proposés sur consultation ont été important

\footnotetext{
3 Traduction de: "Toda experiência na escola, seja ela de sucesso ou de fracasso, fará parte do processo de desenvolvimento do aluno" (LIMA, 2013, p. 4).

4 Voir : <https://www.acfas.ca/app/congres/d/programme/colloques/6262>
} 


\section{Reuista Letras Racas}

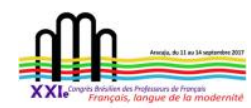

Actes du XXlème Congrès Brésilien des Professeurs de Français dans Édition spéciale de la Revue Letras Raras, 2021 ISSN : 2317-2347 - v. 10, Dossier Spécial (2021)

Todo o conteúdo da RLR está licenciado sob Creative Commons Atribuição 4.0 Internacional

également comme l'une des possibilités d'évaluer à travers des tests. Différencier test d'examen est aussi primordial.

Nous constatons que l'enseignante était la lectrice privilégiée de la production réalisée par les apprenants. Selon les chercheurs en évaluation, l'enseignant est partenaire dans ce processus d'évaluation (Villas Boas, 2002, 2008, 2017, 2019). La rétroaction de l'écriture et les commentaires ont eu une valeur fondamentale dans l'apprentissage des structures de la morphosyntaxique puisque tout se faisait dans la langue-cible. L'enseignante et les apprenants communiquaient à travers l'écriture et réécriture de façon hebdomadaire.

L'évaluation formative est procédurale. Lors du second semestre de 2016, les étudiants ont comparé les différentes façons d'évaluer et le fait d'avoir une évaluation formative n'avait pas posé de problèmes lors de la grève quand les étudiants étaient mobilisés pour des activités d'occupation des espaces de l'Université. Ils ont compris que l'évaluation est procédurale et non sommative, soi-disant, un seul produit à la fin du semestre les aiderait à mieux surmonter ce moment de revendication étudiantes. Les apprenants ont témoigné à plusieurs reprises leurs expériences diverses d'évaluation lors de cette période de grève.

Nous comprenons que ces étudiants n'ont pas reconnu le portfolio comme une pratique d'évaluation formative au départ mais qu'à la fin du semestre ils ont exprimé leur satisfaction à l'accomplir et que la pratique d'écriture quotidienne : a) a facilité le dialogue et la communication enseignant/apprenant ; b) a induit des moments de réflexions en classe sur la langue française, sa structure et sur la pratique d'évaluation formative en cours de langues. Ce qui était certain : le portfolio laissait des traces et des possibilités à de futurs dialogues.

\section{Références}

AMBRÓSIO, M. O uso de portfolio no Ensino Superior. 2ª ed. Petrópolis: Vozes, 2013.

BUGNARD, P.-P. L'autoévaluation dans l'espace historique de deux maîtresses infidèles : la note dans la classe. In : COHEN, P.-F. ; BÉLAIR, L. M. Évaluation et autoévaluation. Quels espaces de formation ? Louvain-la-Neuve : DEBOECK, 2015, p. 15-36.

CASTELLOTTI, V. L'école française et les langues des enfants : quelle mobilisation de parcours plurilingues et pluriculturels ? In: CHISS, J.-L (sous la direction). Immigration, École et didactique du français. Paris : Les Editions Didier, 2008, p. 231-279. 

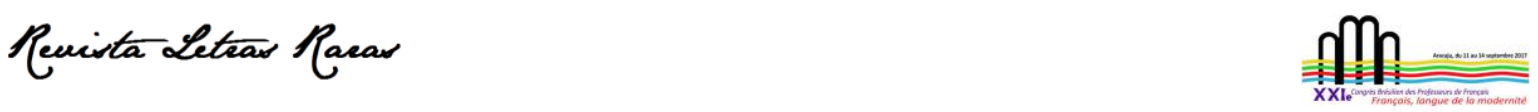

Actes du XXlème Congrès Brésilien des Professeurs de Français dans Édition spéciale de la Revue Letras Raras, 2021 ISSN : 2317-2347 - v. 10, Dossier Spécial (2021)

Todo o conteúdo da RLR está licenciado sob Creative Commons Atribuição 4.0 Internacional

CHANTAL, N. Synthèse réflexive. 1998. Disponible sur :

<http://www.tact.fse.ulaval.ca/fr/html/nathalie/stage/synthese.htm> Accès le 09 aout 2021.

CORACINI, M. J. R. F. Discurso e Escrit(ur)a de si: entre a necessidade e a (im)possibilidade de ensinar. In: ECKERT-Hoff, B. M.; CORACINI, M. J. R. F.. (orgs). Escrit(ur)a de si e alteridade no espaco papel-tela: alfabetização, formação de professores, línguas materna e estrangeira. Campinas, SP: Mercado de Letras, 2010, p. 17-50.

CUQ, J.-P.; GRUCA, I. Cours de Didactique du Français Langue Étrangère et Seconde. Paris: Presses Universitaires de Grenoble, $4^{\mathrm{a}}$ ed., 2017.

HEDID, S. La formation des nouveaux enseignants de FLE à l'évaluation de l'écrit. Discours sur une pratique enseignante. In: Dialogues et Cultures, 61. La formation initiale des enseignants de français langue étrangère. FIPF. Louvain-la-Neuve: EME Éditions, p. 109-117, 2017.

HESS, R. Le Journal des Idées. Paris : Presses Universitaires de Saint-Gemme, 2005.

LIMA, E. S. Avaliação na Escola. São Paulo, SP: Editora Sobradinho, 2013.

TARDIF, M., BORGES, C., MALO, A. Le virage réflexif en éducation : Où en sommes-nous 30 ans après Schön ? Bruxelles : Editora De Boeck Supérieur [Pédagogie en développement], 2012.

VANHULLE, S. Un "genre réflexif" pour travailler avec des savoirs hétérogènes. In: ETIENNE, R. at al. (sous la direction de). L'Université peut-elle vraiment former les enseignants? Quelles tensions? Quelles modalités? Quelles conditions? Bruxelles: Editions De Boeck, p. 201-213, 2009.

VELTCHEFF, C.. Évaluer par les tâches ; une évaluation formative accessible aux enseignants et aux apprenants. In : Le Français dans le monde. Recherches et applications : La perspective actionnelle et l'approche par les tâches en classe de langue. Paris : CLE International, 2009, pages 133-143.

VILLAS BOAS, B. M. de F. Construindo a avaliação formativa em uma escola de Educação Infantil e Fundamental. In: VILLAS BOAS, B. M. F. (Org.). Avaliação: políticas e práticas. Campinas - SP: Papirus, 2002, v. 01, p. 113-143. [Coleção Magistério: formação e trabalho pedagógico].

VILLAS BOAS, B. M. de F. Portfólio, Avaliação e Trabalho pedagógico. Campinas - SP: Papirus, 2005.

VILLAS BOAS, B. M. de F. Virando a Escola do Avesso por Meio da Avaliação. - Campinas, SP: Papirus, 2008.

VILLAS BOAS, B. M. de F. (Org.). Avaliação: Interações com o trabalho pedagógico. - Campinas, SP: Papirus, 2017.

VILLAS BOAS, B. M. de F. (Org.). Conversas sobre avaliação. - Campinas, SP: Papirus, 2019. 